\title{
Acute Pancreatitis: New Developments and Strategies for the Hospitalist
}

\author{
John F. Dick, III, MD ${ }^{1 *}$, Timothy B. Gardner, MD, MS², Edward J. Merrens, MD, MS
}

${ }^{1}$ Geisel School of Medicine, Section of Hospital Medicine, Dartmouth-Hitchcock Medical Center, Lebanon, New Hampshire; ${ }^{2}$ Geisel School of Medicine, Section of Gastroenterology and Hepatology, Dartmouth-Hitchcock Medical Center, Lebanon, New Hampshire.

Acute pancreatitis (AP) remains the most common reason for hospital admission of all the gastrointestinal illnesses in the United States. Since the last narrative review in the Journal of Hospital Medicine in 2010, new developments in regard to diagnosis and classification, fluid resuscitation, antibiotic use, nutritional support, and management of complications have helped refine the approach and improve outcomes in this disease. Whereas there is still no proven pharmacologic therapy to specifically combat the inflammatory consequences of $\mathrm{AP}$, recent interventions have led to increased survival, shorter length of stay, and more appropriate transfer criteria for pancreatitis patients. This case-oriented review will highlight these developments and emphasize the primary role of the hospitalist in managing AP over the course of the admission. It will focus on when to coordinate with subspecialists, how to deliver effective yet efficient hospitalized care, and how to optimize appropriate discharge planning. Journal of Hospital Medicine 2016;11:724-729. (C) 2016 Society of Hospital Medicine
A 55-year-old man presents with colicky right upper quadrant pain radiating to his back for 12 hours. He does not use ethanol and has no familial or personal history of pancreatic disease. Pertinent laboratory values include: white blood cell count $23.610^{3} / \mu \mathrm{L}$; hemoglobin $16.2 \mathrm{~g} / \mathrm{dL}$; blood urea nitrogen (BUN) 52 $\mathrm{mg} / \mathrm{dL}$; aspartate aminotransferase $110 \mathrm{U} / \mathrm{L}$; alanine aminotransferase $272 \mathrm{U} / \mathrm{L}$; alkaline phosphatase 432 $\mathrm{U} / \mathrm{L}$; total bilirubin $4.3 \mathrm{mg} / \mathrm{dL}$; amylase $2230 \mathrm{U} / \mathrm{L}$; lipase $1623 \mathrm{U} / \mathrm{L}$. He is afebrile, normotensive, and not hypoxic, but his respiratory rate is 30 . He has voluntary guarding with palpation of the abdomen, decreased bowel sounds, and decreased breath sounds at the left lung base. A transabdominal ultrasound of the right upper quadrant reveals cholelithiaisis without choledocholithiasis. There is mild peripancreatic stranding and the head is slightly edematous.

\section{NATURAL HISTORY}

Acute pancreatitis (AP) is a common cause for emergency room presentation, resulting in over 280,000 hospital admissions in the United States at a cost of nearly $\$ 3$ billion dollars annually. ${ }^{1}$ In its mildest form it may require a 2- to 5-day hospital stay and an uncomplicated discharge. In more severe cases, such as in the setting of pancreatic necrosis and/or the development of organ failure, hospitalization can

\footnotetext{
*Address for correspondence and reprint requests: John F. Dick, MD, Infectious Section of Hospital Medicine, Dartmouth-Hitchcock Medical Center, One Medical Center Drive, Lebanon, NH 03756; Telephone: 603650-8380; Fax: 603-650-6110; E-mail: john.f.dick.iii@hitchcock.org

Additional Supporting Information may be found in the online version of this article.

Received: January 12, 2016; Revised: April 1, 2016; Accepted: April 24, 2016

2016 Society of Hospital Medicine DOI 10.1002/jhm.2615

Published online in Wiley Online Library (Wileyonlinelibrary.com).
}

feature a much longer and complicated hospital course. $^{2}$

\section{DIAGNOSTIC CRITERIA AND CLASSIFICATION}

AP is diagnosed by the patient having 2 out of the following 3 criteria: (1) classic clinical symptoms with abdominal pain consistent with AP (2) serum amylase and/or lipase greater than 3 times the upper limit of normal, and/or (3) characteristic findings from abdominal imaging. ${ }^{3}$ It is important for the hospitalist to recognize that patients can have AP with normal serum amylase and/or lipase levels, as long as their clinical symptoms and imaging exam are consistent with the disease. ${ }^{4}$ It is also important to recognize that amylase and/or lipase elevation is not $100 \%$ specific for pancreatitis; alternate conditions that elevate amylase levels include renal insufficiency, intestinal ischemia and obstruction, macroamylasemia, and multiple medications, whereas lipase elevations can be seen in spontaneous bacterial peritonitis, intestinal ischemia, and esophagitis. ${ }^{5}$

AP is classified as either mild (absence of organ failure or local complications), moderate (local complications and/or transient organ failure $<48$ hours) or severe (persistent organ failure $>48$ hours). ${ }^{3}$ Organ failure is defined by the modified Marshall score, and local complications include pancreatic fluid collections, pancreatic and peripancreatic necrosis, and vascular thrombosis (Table 2). ${ }^{6}$

\section{ETIOLOGY}

Transiently obstructing gallstones, thought to account for about $50 \%$ of cases, are the most common cause of AP. The rising prevalence of obesity, which is a known risk factor for AP due to the corresponding increase in the frequency of gallstones, suggests that this will continue to be the leading cause going 
TABLE 1. Major Updates in Management of AP in the Last Five Years

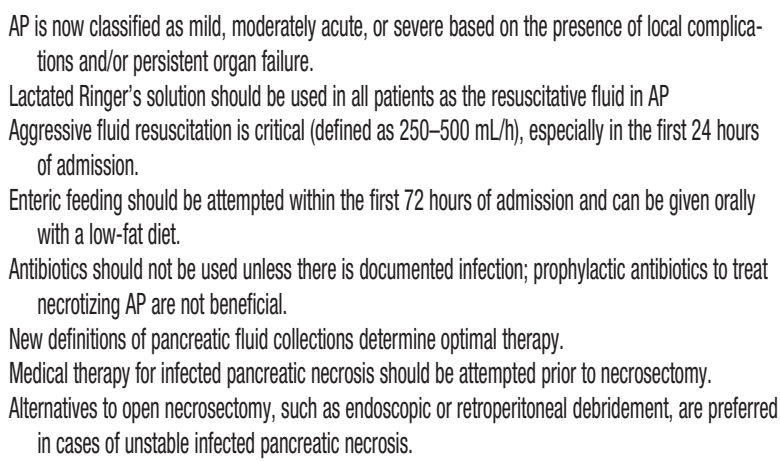

NOTE: Abbreviations: AP, acute pancreatitis.

forward. ${ }^{7}$ Alcohol use is associated with both acute and chronic pancreatitis; however, the extent to which it is a primary cause of AP is uncertain. ${ }^{8}$ Trauma, medications, hypercalcemia, and hypertriglyceridemia must also be considered; however, they are much less common. AP from endoscopic retrograde cholangiopancreatography (ERCP) occurs following 5\% of procedures and from endoscopic ultrasound (EUS) fineneedle aspiration following $1 \%$. Although several medications are clearly associated with AP, many that were previously invoked seem less likely. ${ }^{9}$ Immunoglobulin G (IgG) 4-related systemic disease, although rare, is becoming more recognized and should be considered when the more common etiologies are ruled out. Finally, it is controversial whether anatomic findings such as pancreatic divisum and functional disorders such as sphincter of Oddi dysfunction cause AP. ${ }^{10}$

Identifying the cause of an acute episode remains important, as subsequent treatment strategies can be tailored to help prevent recurrence. A thorough personal history, including prior gallbladder disease, alcohol use, and medications is strongly recommended. Basic laboratory studies including liver function tests, serum calcium and triglycerides, as well as a right upper quadrant ultrasound are indicated in all patients presenting with AP. ${ }^{1}$ Idiopathic AP is not uncommon. Given the increasing awareness of genetic factors, potential role of advanced endoscopy, and higher risk of recurrence in this group, patients with idiopathic AP should be referred to specialized centers of expertise. ${ }^{4}$

\section{PROGNOSTICATION}

Most cases of AP are mild and do not require prolonged hospitalization; however, because $5 \%$ of hospitalized patients will die from this disease, prognostic criteria are needed to determine high-risk cases. ${ }^{11}$ Multiple systems have been developed (Bedside Index for Severity in Acute Pancreatitis, Ranson's, Acute Physiology and Chronic Health Evaluation II, Computed Tomography Severity Index), but all have had difficulty achieving accuracy in a user-friendly tool; because of this, hospitalists should instead focus on the individual laboratory parameters that correlate with pathophysiologic derangement. Elevations in BUN and hematocrit indicate hypovolemia, leukocytosis, and fluid sequestration are indicators of the inflammatory cascade. Creatinine, elevated liver tests, and hypoxia are indicators of organ damage. Low calcium is reflective of fat necrosis saponification (endorgan damage) and also an indicator of hypovolemia. Essentially, the prediction of severity depends on identifying indications of end-organ damage in a timely manner and can be performed through a combination of age, known comorbidities, physical exam, and basic laboratory testing. ${ }^{12}$

\section{ADDITIONAL INITIAL IMAGING}

Although sensitive and specific for AP, routine computed tomography $(\mathrm{CT})$ imaging for all patients presenting with suspected AP is not indicated. The diagnosis is often clear on a clinical and lab basis alone, and most patients with AP will improve within 48 hours. ${ }^{13}$ CT or magnetic resonance imaging (MRI) can be considered for patients with an unclear diagnosis and indeterminate ultrasound or in those who are not improving within the first 48 to 72 hours after presentation. This additional imaging can help make an alternative diagnosis or detect an early complication such as pancreatic necrosis. CT is preferred; however, MRI may be utilized if there is a high suspicion for biliary stones that were not seen on ultrasound or when CT is indicated but impaired renal function precludes its use. ${ }^{4}$ In patients presenting with recurrent idiopathic AP, EUS is recommended to evaluate for an occult malignancy or microlithiasis. ${ }^{14}$

\section{INITIAL CLINICAL MANAGEMENT}

Without evidence of either (1) ascending cholangitis or (2) proven choledocholithiasis with clinical decompensation and worsening liver tests, ERCP should not

TABLE 2. Modified Marshall Scoring System for Organ Dysfunction

\begin{tabular}{|c|c|c|c|c|c|}
\hline Organ System Score & 0 & 1 & 2 & 3 & 4 \\
\hline Respiratory $\left(\mathrm{PaO}_{2} / \mathrm{FiO}_{2}\right)$ & $>400$ & $301-400$ & $201-300$ & $101-200$ & $<101$ \\
\hline Renal serum creatinine (mg/dL) & $<1.4$ & $1.4-1.8$ & $1.9-3.6$ & $3.7-4.9$ & $>4.9$ \\
\hline Cardiovascular systolic blood pressure ( $\mathrm{mm} H \mathrm{Hg}$ ) & $>90$ & $<90$, fluid responsive & $<90$, not fluid responsive & $<90, \mathrm{pH}<7.3$ & $<90, \mathrm{pH}<7.2$ \\
\hline
\end{tabular}


be performed and management should be focused on supportive care, pain control, and monitoring prognostic information regarding severity. The initial management of AP should include fluid replacement with lactated Ringer's (LR) solution at $5-10 \mathrm{~mL} / \mathrm{kg} / \mathrm{h}$ to achieve noninvasive parameters of a heart rate $<120$, mean arterial pressure 65 to $85 \mathrm{~mm} \mathrm{Hg}$, and urine output $>0.5$ to $1 \mathrm{~mL} / \mathrm{kg} / \mathrm{h}$. LR decreases the incidence of the systemic inflammatory response syndrome (SIRS) by $80 \%$ compared with normal saline., ${ }^{4,15}$ Early and sufficient fluid replacement is associated with decreased rates of SIRS and organ failure, whereas under-resuscitation has been associated with necrosis and increased mortality. In the first 48 to 72 hours of admission, frequent assessment of hemoglobin $(\mathrm{HgB})$ and BUN, as well as urine output measurements, should be obtained to make sure fluid resuscitation is adequate. ${ }^{4}$ Intravenous fluid replacement should continue in the hospital until the patient can adequately maintain appropriate fluid intake orally. Prophylactic antimicrobial therapy is not indicated in initial cases of AP, unless there are clear signs of an underlying infection. Pain control is essential, and efforts at reintroducing oral feeding should be initiated once the pain is decreasing. There are no randomized trials that have identified an optimal narcotic-based pain regimen. On a daily basis, a complete blood count, renal function, and liver function should be measured. There is no reason to continue measuring serum amylase or lipase, as it may not be elevated in some instances in AP, and its fluctuation is not indicative of a change in clinical status.

\section{Case Management Strategy}

The patient has mild AP based on lack of organ failure and local complications and is admitted to the regular medical floor. The etiology appears to be due to cholelithiasis, but the patient does not have cholangitis, so ERCP was not considered, and antibiotics were not started. Aggressive fluid resuscitation with lactated Ringer's is started at a rate of $350 \mathrm{~mL} / \mathrm{h}$, and BUN and $\mathrm{HgB}$ are monitored every 8 hours to make sure that these levels are decreasing. The patient is placed on a low-fat diet and encouraged to eat as tolerated. Further imaging is not ordered at this time.

\section{Hospital Day 3}

The patient's liver tests have normalized, but the BUN continues to rise $(82 \mathrm{mg} / \mathrm{dL})$ despite aggressive fluid resuscitation with LR. He remains afebrile and normotensive, but is now hypoxic and requiring nasal cannula oxygen at $4 \mathrm{~L} / \mathrm{min}$ to maintain his oxygen saturation above $90 \%$. His abdominal pain is controlled with intravenous opiates, but he is not hungry or able to eat. With these changes in his clinical course, a CT scan is performed, which demonstrates acute peripancreatic necrosis centered on the head of the pancreas.

\section{PERSISTENT ORGAN FAILURE AND PANCREATIC NECROSIS}

Generally, patients with severe AP (persistent organ failure $>48$ hours following admission) should be followed in the intensive care unit for effective monitoring and support.

Pancreatic necrosis is defined as a diffuse or focal area of nonviable pancreatic parenchyma $>3 \mathrm{~cm}$ in size or $>30 \%$ of the pancreas. ${ }^{1}$ Extrapancreatic necrosis can also be present, and is associated with adverse outcomes such as organ failure. ${ }^{16}$ Pancreatic and extrapancreatic necrosis can be sterile or infected. The presence of infection does not necessarily increase the risk of subsequent organ failure.

\section{FEEDING}

In patients with mild pancreatitis, oral feeding with a low-fat solid diet can be initiated when nausea, vomiting, and pain have resolved. ${ }^{1}$ A randomized controlled trial demonstrated that patients who receive oral feeding earlier in the course of their stay have a shorter length of stay and fewer complications. ${ }^{17}$ In patients with evolving AP who unable to tolerate oral feeding, enteral tube feeding either via nasogastric or nasojejunal routes should be initiated to support the intestinal biome and prevent bacterial translocation from the gut to the pancreas. Nasogastric feeding appears to be as safe as nasojejunal feeding. ${ }^{18}$ Parenteral nutrition should only be used as a second-line therapy if adequate caloric requirements cannot be maintained via an enteral route given the increased rate of infections and mortality when compared with nasoenteric feeding. ${ }^{19}$ The most recent study on when to start enteric feeding in patients at high risk for complications demonstrates no benefit from starting nasoenteric feeding within the first 24 hours of admission compared to starting an oral diet at 72 hours. ${ }^{20}$

\section{INTRA-ABDOMINAL COMPARTMENT SYNDROME}

A sometimes overlooked consequence of aggressive fluid resuscitation can be the development of intraabdominal compartment syndrome, which is defined as new organ dysfunction with concomitant intraabdominal pressure measurements $>20 \mathrm{~mm} \mathrm{Hg}$. Patients with an increasingly tense abdomen, oliguria, or increasing ventilator requirements should have intravesical pressures measured with a urinary catheter. Initial treatment consists of decreasing the fluid resuscitation rate along with supportive measures such as reducing ventilator tidal volume and placing nasogastric and rectal tubes; if not successful, surgical decompression is indicated.

\section{SUBSPECIALIST INVOLVEMENT}

The majority of mild AP cases can effectively be managed by hospitalists, and there is no evidence that subspecialist involvement improves important clinical 


\begin{tabular}{|c|c|c|}
\hline Type Of Collection & Description & Optimal Management \\
\hline Acute Fluid collection (AFC) & $\begin{array}{l}\text { Fluid collection that develops less } \\
\text { than } 4 \text { weeks since initial injury, } \\
\text { contains no solid debris and has no } \\
\text { encapsulated wall. }\end{array}$ & $\begin{array}{l}\text { Delay intervention as most will } \\
\text { resolve spontaneously. }\end{array}$ \\
\hline Pancreatic & $\begin{array}{l}\text { Collection that arises from a } \\
\text { mature acute fluid collection and is } \\
\text { surrounded by a well-defined wall } \\
\text { and contains essentially no solid } \\
\text { debris. }\end{array}$ & $\begin{array}{l}\text { Delay intervention as most will } \\
\text { resolve spontaneously. If no } \\
\text { resolution, perform minimally } \\
\text { invasive procedure to drain. }\end{array}$ \\
\hline tion (ANC) & $\begin{array}{l}\text { Collection that arises in the } \\
\text { context of pancreatic or } \\
\text { peripancreatic necrosis that } \\
\text { develops less than } 4 \text { weeks since } \\
\text { the initial injury. }\end{array}$ & $\begin{array}{l}\text { Delay intervention a minimum of } \\
4 \text { weeks. }\end{array}$ \\
\hline Walled-Off Necrosis (WON) & $\begin{array}{l}\text { Mature collection of solid and } \\
\text { liquid debris that arises from an } \\
\text { acute necrotic collection and has a } \\
\text { well encapsulated wall. }\end{array}$ & $\begin{array}{l}\text { Perform minimally invasive } \\
\text { necrosectomy (endoscopic, } \\
\text { laparoscopic, retroperitoneal). }\end{array}$ \\
\hline
\end{tabular}

FIG. 1. Classification and management of pancreatic fluid collections.

outcomes in mild disease. The need for subspecialty input should be based on the need for a procedure such as ERCP or collaborative care if the patient develops more acute complications requiring ongoing critical care support or decisions centered on sampling of fluid collections and/or necrosectomy.

\section{Case Management Strategy}

The patient is transferred to the intensive care unit for closer monitoring of his hemodynamic and respiratory status. His LR is held at $250 \mathrm{~mL} / \mathrm{h}$ and his BUN is checked every 8 hours. He undergoes serial abdominal exams and twice-daily bladder pressure measurements to evaluate for intra-abdominal compartment syndrome. Antibiotics continue to be held as there is no evidence of pancreatic or extrapancreatic infection. A nasogastric tube is placed and enteral feeding begun with a low-fat formulation and advanced as tolerated. The gastroenterology service is consulted to assist in management.

\section{Hospital Day 17}

With optimal intensive care unit monitoring of fluid status, early initiation of enteral feeding, and manage- ment of pain, the patient's vital signs have normalized and is he is transferred to the medical ward and is tolerating a clear liquid diet. In the next 48 hours, he becomes febrile. Urinalysis is unremarkable and blood cultures show no growth. Given continued fevers without a clear source, a CT scan of the abdomen is obtained. It demonstrates formation of a necrotic collection.

\section{DEFINITION AND MANAGEMENT OF PANCREATIC FLUID COLLECTIONS}

There are 4 main types of pancreatic collections, which include acute fluid collections, acute necrotic collections, pseudocysts, and walled off necrosis (Figure 1). ${ }^{3}$ Acute fluid collections (AFC) develop less than 4 weeks after an episode of interstitial pancreatitis. They are found in the pancreatic parenchyma or peripancreatic tissue and usually resolve without requiring intervention. When a fluid collection develops in the context of pancreatic necrosis, it is known as an acute necrotic collection. If an AFC does not resolve in 4 weeks and develops an encapsulated wall that lacks solid debris, it is characterized as a pseudocyst. Pseudocysts are usually extrapancreatic, but 
occasionally can be intrapancreatic as a result of a disrupted pancreatic duct. Walled off necrosis (WON) occurs after 4 weeks, contains solid debris, and occurs only in the context of necrotizing pancreatitis.

The most important strategy for the hospitalist in managing AFC is to delay intervention as long as possible. ${ }^{14,21,22}$ This decision generally requires multidisciplinary input (for example with gastroenterology, surgical, and infectious diseases consultative services), as any intervention performed prematurely may lead to significant morbidity and occasional mortality. The vast majority of AFCs and pseudocysts will resolve spontaneously. In addition, most ANCs can be allowed to mature beyond the time of the initial hospitalization and can be managed as an outpatient if/ when they proceed to WON.

\section{INFECTED PANCREATIC NECROSIS}

In the last decade, the paradigm for managing infected pancreatic necrosis has shifted dramatically. It is no longer necessary to sample the pancreas to make the diagnosis of infected pancreatic necrosis. In most cases, a careful history, clinical examination, and imaging should be able to make the diagnosis. ${ }^{1,23}$ Historically, open necrosectomy/debridement was the standard for the treatment of infected necrosis, but due to increased mortality, this practice has been abandoned. Currently, it is recommended that in stable patients, a course of pancreas-penetrating antibiotics (such as meropenem) can be tried to allow for better organization of the inflammatory reaction. Subsequently, if the patient remains ill and the infected necrosis has not resolved, minimally invasive necrosectomy, via a variety of techniques such as endoscopy, laparoscopy, or a video-assisted retroperitoneal approach, should be employed before considering any open surgery. Minimally invasive techniques have the advantages of not only being as successful as open surgery, but also have lower complication rates. ${ }^{24}$

\section{Case Management Strategy}

In the setting of fevers and a necrotic fluid collection, the patient is empirically started on meropenem. The pancreatic fluid collection has caused pressure on the stomach, which has led to nausea and vomiting, but he has tolerated continued enteral feeding via a nasogastric tube.

\section{Hospital Day 29}

The patient undergoes successful direct endoscopic necrosectomy on hospital day 29 after a repeat CT scan demonstrates complete maturation of the walled off pancreatic necrosis. Following the procedure, his nausea resolves and he is able to tolerate transition to a low-fat diet.

\section{OTHER COMPLICATIONS}

Prior to discharge, it is important to consider other possible complications that may have arisen. New onset glucose intolerance or diabetes, thrombosis of the portal vasculature, and/or splenic aneurysm development can all occur several weeks into the hospitalization. The hospitalist must be aware of clinical clues such as new-onset ascites due to thrombosis of the superior mesenteric vein.

\section{PREVENTING READMISSIONS}

Patients presenting with acute pancreatitis have a 30day readmission rate around $20 \% .^{25}$ Prognostic factors that reduce the risk of readmission include patient tolerating a solid diet, absence of other gastrointestinal symptoms (nausea, vomiting, or diarrhea), and well-controlled pain. The presence of pancreatic necrosis and the necessity for antimicrobial therapy increase the risk of readmission. ${ }^{25}$ In terms of modifiable risk factors, risk of readmission has been correlated with alcohol as etiology of index hospitalization and tobacco abuse. Careful attention to addressing alcohol use and abuse as well as the challenging transition from acute to chronic pain control for patients with chronic pancreatitis is essential, as it is often recurrent pain and possibly not pancreatitis per se that may be the most common reason for hospital readmission. Finally, cholecystectomy for biliary AP should be performed prior to discharge; if this is not feasible, short-interval outpatient follow-up for surgery is imperative.

\section{Management Strategy}

The patient undergoes an uneventful laparoscopic cholecystectomy on hospital day 35 . He is discharged to a skilled nursing facility with physical and occupational rehabilitation services. He has follow-up scheduled with the gastroenterology service in 2 weeks. His case highlights many of the potential complications of acute pancreatitis and the major updates to management of this common illness (Table 1).

Disclosure: Nothing to report.

\section{References}

1. Tenner S, Baillie J, DeWitt J, et al. American college of gastroenterology guideline: management of acute pancreatitis. Am J Gastroenterol. 2013;108:1400-1415.

2. Johnson CD, Besselink MG, Carter R. Acute pancreatitis. BMJ. 2014; 349:g4859.

3. Banks PA, Bollen TA, Dervenis C, et al. Classification of acute pancreatitis-2012: revision of Atlanta classification and definitions by international consensus. Gut. 2013;62:102-111.

4. Wu BU, Banks PA. Clinical management of patients with acute pancreatitis. Gastroenterology. 2013;144:1272-1281.

5. Clavien PA, Robert J, Meyer P, et al. Acute pancreatitis and normoamylasemia. Not an uncommon combination. Ann Surg. 1989;2010: 614-620.

6. Shah AM, Eddi R, Kothari ST, et al. Acute pancreatitis with normal serum lipase: a case series. JOP. 2010;11:369-372.

7. Hong S, Qiwen B, Ying J, et al. Body mass index and the risk and prognosis of acute pancreatitis: a meta-analysis. Eur J Gastroenterol Hepatol. 2011;23(12):1136-1143.

8. Herreros-Villanueva M, Hijona E, Bañales JM, et al. Alcohol consumption on pancreatic diseases. World J Gastroenterol. 2013;19(5): 638-647.

9. Nitsche C, Maertin S, Scheiber J, et al. Drug-induced pancreatitis. Curr Gastroenterol Rep. 2012;14:131-138. 
10. DiMagno MJ, Dimagno EP. Pancreas divisum does not cause pancreatitis, but associates with CFTR mutations. Am J Gastroenterol. 2012; 107:318-320.

11. Mofidi R, Duff MD, Wigmore SJ, et al. Association between early systemic inflammatory response, severity of multiorgan dysfunction and death in acute pancreatitis. Br J Surg. 2006;93:738-744.

12. Banks PA, Freeman ML. Practice guidelines in acute pancreatitis. Am J Gastroenterol. 2006;101(10):2379-2400.

13. Yadav D, O 'Connell M, Papachristou GI. Natural history following the first attack of acute pancreatitis. Am J Gastroenterol. 2012;107: 1096-1103.

14. Working Group IAP/APA Acute Pancreatitis Guidelines. IAP/APA Evidence-based guidelines for the management of acute pancreatitis. Pancreatology. 2013;13:e1-e15.

15. Freeman ML, Werner J, van Santvoort HC, et al. Interventions for necrotizing pancreatitis: summary of a multidisciplinary consensus conference. Pancreas. 2012;41(8):1176-1194.

16. Bakker OJ, van Santvoort H, Besselink M, et al. Extrapancreatic necrosis without pancreatic parenchymal necrosis: a separate entity in necrotising pancreatitis? Gut. 2013;62(10):1475-1480.

17. Petrov MS, Kukosh MV, Emelyanov NV. A randomized controlled trial of enteral versus parenteral feeding in patients with predicted severe acute pancreatitis shows a significant reduction in mortality and in infected pancreatic complications with total enteral nutrition. Dig Surg. 2006;23:336-345.
18. Singh N, Sharma B, Sharma M, et al. Evaluation of early enteral feeding through nasogastric and nasojejunal tube in severe acute pancreatitis. A non-inferiority randomized controlled trial. Pancreas. 2012; 41:153-159.

19. Al-Omran M, Albalawi ZH, Tashkandi MF, Al-Ansary LA. Enteral versus parenteral nutrition for acute pancreatitis. Cochrane Database Syst Rev. 2010;1:CD002837.

20. Bakker OJ, van Brunschot S, van Santvoort HC, et al. Early versus ondemand nasoenteric tube feeding in acute pancreatitis. N Engl J Med. 2014;371(21):1983-1993.

21. Van Santvoort HC, Bakker OJ, Bollen TL, et al. A conservative and minimally invasive approach to necrotizing pancreatitis improves outcome. Gastroenterology. 2011;141:1254-1263.

22. Van Santvoort HC, Besselink MG, Bakker OJ, et al. Endoscopic necrosectomy in necrotizing pancreatitis: indication is the key. Gut. 2010;59:1587.

23. Goldenberg DE, Gordon SR, Gardner, TB. Management of acute pancreatitis. Expert Rev Gastroenterol Hepatol. 2014;8(6):1-8.

24. Whitehead DA, Gardner TB. Evidence-based management of acute pancreatitis. Curr Treat Options Gastroenterol. 2014;9(2): $175-180$.

25. Whitlock TL, Tignor A, Webster EM, et al. A scoring system to predict readmission of patients with acute pancreatitis to the hospital within thirty days of discharge. Clin Gastroenterol Hepatol. 2011;9(2): 175-180. 